\title{
On-Line Monitoring of Cable Trench and Cable Pit by Using Metal Armored FBG Temperature Sensors
}

\author{
Zhen Wang, ${ }^{\mathrm{a}, \mathrm{c}}$, Min Cao ${ }^{\mathrm{b}}$, Da-Da Wang ${ }^{\mathrm{b}}$, Shao-Quan Zhang ${ }^{\mathrm{b}}$, Chuan $\mathrm{Li}^{\mathrm{c} *}$, \\ Ying-Na $\mathrm{Li}^{\mathrm{c}}$, Xiao-Long $\mathrm{Li}^{\mathrm{a}, \mathrm{c}}$ \\ ${ }^{a}$ Graduate Workstations, Kunming University of Science and Technology \& Yunnan Power Grid Corp Kunming 650217, China \\ ${ }^{b}$ Electric Power Research Inst., Yunnan Prov. Electric Power Test \& Research Inst. (Group) Co., Ltd, Kunming 650217, China \\ ${ }^{c}$ Faculty of Information Engineering and Automation, Kunming University of Science and Technology, Kunming 650500, China
}

\begin{abstract}
Substation cable trench and cable pit lay many cables, which are used to connect electrical equipment. The longrunning of cable will probably cause insulation material aging, leakage sparking and local overheating occur, which eventually lead to a cable fire, the temperature of cable surface usually reflected the situation of the cable. Metal armored fiber Bragg grating temperature sensor are applying in a substation cable trench and cable pit, with four fiber trench it monitor the 60 cable temperature measuring point in current time. The 24-hours real-time monitoring indicates that the temperature of morning is the minimum, and the temperature of afternoon is the maximum. The temperature of cable surface ranges in the similar curve with ambient temperature. In the long-term of 479 days, the minimum of the average daily temperature range of 60 sensors is $21.24^{\circ} \mathrm{C}$,detected at number 9 trench 1 , The maximum is $37.43^{\circ} \mathrm{C}$, detected at number 4 trench 2, which reached its maximum temperature $43.41^{\circ} \mathrm{C}$ on July 3 , 2010 , and the minimum temperature is $4.37^{\circ} \mathrm{C}$ on January 17,2011 ,number 13 trench 1 . The temperature of sensors changed in the same trend with seasons.
\end{abstract}

Keywords: Fiber Bragg grating, power cable, on-line monitoring, cable trench, cable pit, environment temperature. ${ }^{a}$

\section{Introduction}

Cable is an important part of electricity system. In the substation, cable is the connection of various electricity device, and links to the control room. It usually lays in cable bridge, cable tunnel, cable interlayer, cable ditch and cable shaft. The plenty of cables, long route, complicated trend and compact arrange. Especially in cable trench and cable pit, a relatively closed, dark and damp environment, cooling condition is poor, the long-term withstand high voltage, large current and heating, lead to the insulation material aging. In addition, as the electric circuit is used for a long time, lacks of regular maintenance and timely update, leakage ignition or local overheating would occur frequently, once the fire area is wide, the repair time is long, there will be a large loss [1], [2]. Temperature of high voltage cable surface reflects inside condition of the cable, whether overload operation or changes of cable operating environment or cable line fault (such as the various fault resulting from partial discharge of main insulation or the destruction of protecting layer) will cause the change of cable surface temperature therefore monitoring of cable surface temperature will timely detect the cable abnormal condition, then we take timely measures to avoid accidents [3], [4].

The wavelength shift of metal armored fiber Bragg grating temperature sensor Bragg is only sensitive to temperature and linearly changes with temperature, we use fiber Bragg grating sensor WDM technology, with 4 fiber trenches to do the timely on-line monitoring of 60 temperature monitoring point

\footnotetext{
* Manuscript received July 14, 2012; revised August 15, 2012.

Corresponding author. Tel.: +86-871-5916597; fax: +86-871-5916597; E-mail address: boatriver@eyou.com.
} 
and environmental temperature of Zhaotong Yanjin substation cable trench and cable pit.

\section{Structure and Operation Principle Of Metal Armored Fiber Bragg Grating Temperature Sensor}

Fiber Bragg grating center wavelength is relative to the core of the effective refractive index $n_{\text {eff }}$ and the grid cycle $\Lambda$, changes of temperature, stress and strain can cause the refractive index $n_{\text {eff }}$ and raster cycle $\Lambda$ change [5], which lead to the center wavelength $\lambda_{B}$ drifts.

Mounted on the cable, fiber Bragg grating temperature sensor inevitably will be squeezed, bending, drawing and stress strain. The temperature measuring of cable fiber Bragg grating temperature sensor should resist traction during the rally and damage from bite of anti rodent animal. Insulating medium covers cable outer part, therefore, to make the sensor sensitive to temperature only and ensure the measuring accuracy, using a metal armored fiber Bragg grating temperature sensor which is not sensitive to external stress, its structure is shown in Fig. 1.

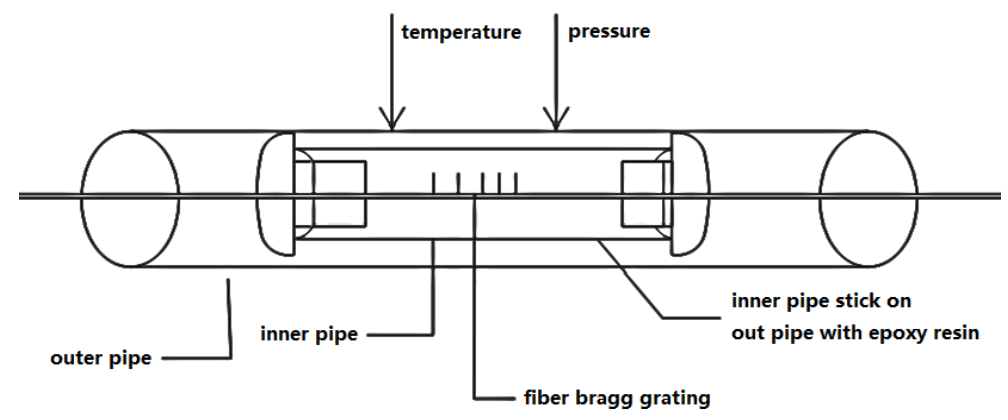

Fig. 1. Structure of metal armored fiber Bragg grating temperature sensor.

In the double layer tube structure, the main function of the outer tube is conducting temperature and eliminating the external stress strain impact on Bragg wavelength offset. As a result of the external stress strain impact, the outer tube longitudinal strain induced grating wavelength value offset is within, we can assume that the grating offset value is very small, and is within the allowable error range, it has little impact on the temperature induced Bragg wavelength offset. The temperature is uniformly transferred to the inner tube. Due to the temperature effect of grating in the inner tube, Bragg wavelength offset value changes, we can get the wavelength offset value by optical fiber AFC port to the spectrum analyzer.

Wavelength variation caused by the change of temperature sensor is shown as in form (1) [6],

$$
\frac{\Delta \lambda_{B}}{\lambda_{B}}=(\alpha+\xi) \Delta T=S_{T} \Delta T
$$

Form (1), $S_{T}$ is temperature coefficient of sensor, $\Delta \lambda_{B}$ is the change of wavelength, $\alpha$ is coefficient of thermal expansion of the fiber, $\xi$ is thermo-optical coefficient.

Therefore, in the elimination of external stress strain field, metal armored fiber Bragg grating temperature sensor wavelength drift changes linearly with temperature, detecting the change of wavelength, so to measure temperature change.

\section{Application of Metal Armored Fiber Bragg Grating Temperature Sensor in Cable Trench and Cable Pits}

The system is used in 220/110/35kV substation cable trench and cable pits of Yunnan power network of Zhaotong Power Supply Bureau Yanjin. When the power cable is installed on the cable trench and cable pits, it is difficult to measure the temperature by manual inspection, so temperature detection system based on the traditional telecommunication transmission can not meet the needs of application. Fiber Bragg grating real-time temperature monitoring is a distributed monitoring system, the position and 
quantity of measuring point can be laid at needs, it holds high precision, fast response, far transmission distance, convenient installation, free of maintenance, harsh environment resistance, electromagnetic interference resistance, intrinsically safe and explosion-proof, has an obvious technical superiority. As shown in Fig. 2.

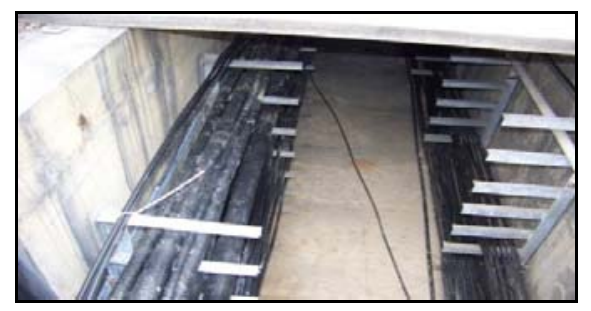

(a)

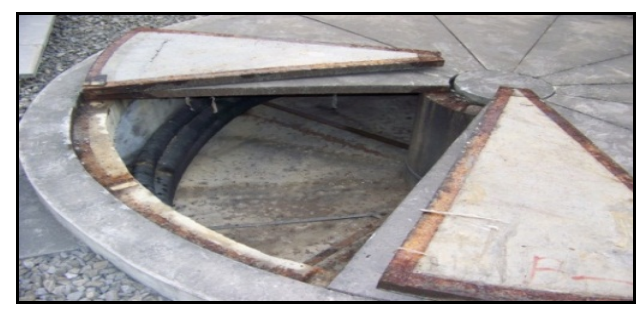

(b)

Fig. 2. (a) Cable Trench; (b) Cable Pit.

The cable temperature rise test has been done in the laboratory, to determine the initial temperature change and temperature distribution of the cable heat fault. Make site design site visits of transformer substation cable trench, cable pits According to a temperature measurement of a 4 core and 30 meters cable, the positioning accuracy is $1 \mathrm{~m}$, temperature resolution is $0.1^{\circ} \mathrm{C}$, the effective measurement distance is up to $4 \mathrm{~km}$. Following the $0.5 \mathrm{M}$ per branch principles, we need 60 metal armored fiber optic Bragg grating temperature sensors in all.

As shown in Fig. 3, the connecting box is placed in the 5 cable well, the 4 core cable communication cable ports, reached to the main control room then leads to No.5 well; 60 metal armored fiber optic Bragg grating temperature sensors are divided into 4 groups, respectively, occupy the $1 \sim 4$ trench of fiber grating analyzer.

The temperature sensors are respectively arranged: $1 \sim 15$ sensors of 1 trench are arranged on the cable well 5, from 5 cable well to $35 \mathrm{kV}$ cable trench high voltage interior, $35 \mathrm{kV}$ cable trench high voltage interior; $1 \sim 15$ sensors of 2 trench are arranged on the cable from the cable pit 5 , cable pit 5 to the cable trench of main control room. 1 15 sensors of 3 trench are arranged on the cable trench from cable pit 3 to cable pit 5, cable pit 3, and the cable trench from cable pit 3 leading up to the main transformer (air cooling control box); $1 \sim 15$ sensors of 4 trench are arranged on the cable pit 1 , cable trench from cable pit 1 to the cable pit 5 .

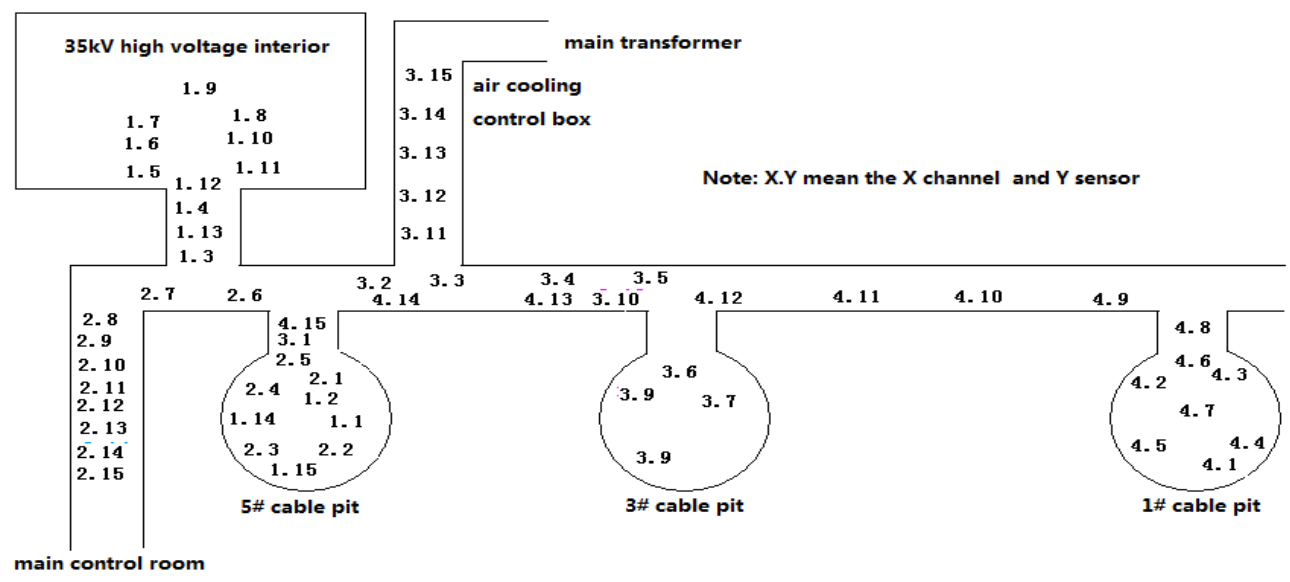

Fig. 3. Cable trench and cable pit sensor layout figure.

\section{On-Line Monitoring Data Analysis of Cable Trench and Cable Pit}

Fig. 4 shows the data change curve of cable trench and cable pit sensors with Yanjin environment temperature in 24 hours, at August 8, 2011. Curves are respectively got in cable trench, high voltage 
interior cable trench, cable pit and the real-time temperature monitoring Zhaotong Yanjin environment data.

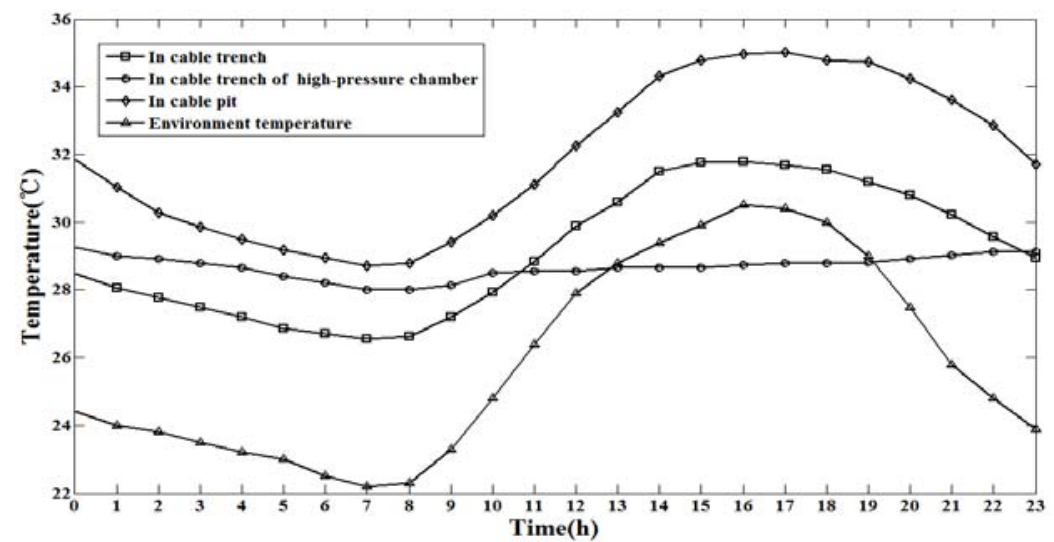

Fig. 4. data change curve of cable trench and cable pit sensors with Yanjin environment temperature in 24 hours

In normal cable conditions of operation, the temperature changes of cable surface are mainly caused by the environment temperature change. Through the Fig. 5 data contrast curve we found that:

The temperature of environment reached its lowest $22.2^{\circ} \mathrm{C}$ at $7: 00$ in the morning, and reached its highest $30.5^{\circ} \mathrm{C}$ at $16: 00$ in the afternoon; The cable trench sensors, reached its lowest $26.54^{\circ} \mathrm{C}$ at $7: 00$, reached its highest $31.78^{\circ} \mathrm{C}$ at $16: 00$; Cable trench sensors of high voltage chamber reached its lowest of $28.01{ }^{\circ} \mathrm{C}$ at $08: 00$, and reached its highest $29.25^{\circ} \mathrm{C}$ at $00: 00$; The cable pit sensors reached its lowest $28.72^{\circ} \mathrm{C}$ at $07: 00$, and reached its highest $35.01^{\circ} \mathrm{C}$ at $17: 00$; The trend of cable surface temperature change and environmental temperature change is consistent, the data consequence is relatively stable.

The temperature of cable pit sensor is $4^{\circ} \mathrm{C}$ higher than that of cable trench on average, The temperature of cable trench sensors of high voltage chamber tend to be flat and is $2^{\circ} \mathrm{C}$ higher than that of cable trench on average at $00: 00$ to $11: 00,3^{\circ} \mathrm{C}$ lower than that of cable trench on average at 12 23. This is because the three teams of sensors laid on different locations, the ventilation performance, closure property of cable pit poorer than that in the trench, the temperature of pit is higher than the trench temperature; High voltage chamber of cable trench is indoor, plenty of equipments, and high quantity of heat production, little effect from external environment temperature, and little temperature change. The temperature sensor effectively monitors the temperature of a day.

Fig. 5 shows the temperature change curve of sensors in cable trench and cable pit in 479 days. Fig. 6 shows the temperature change curve of Yanjin environment in 479 days, the time is from June 6, 2010 to September 28, 2011.

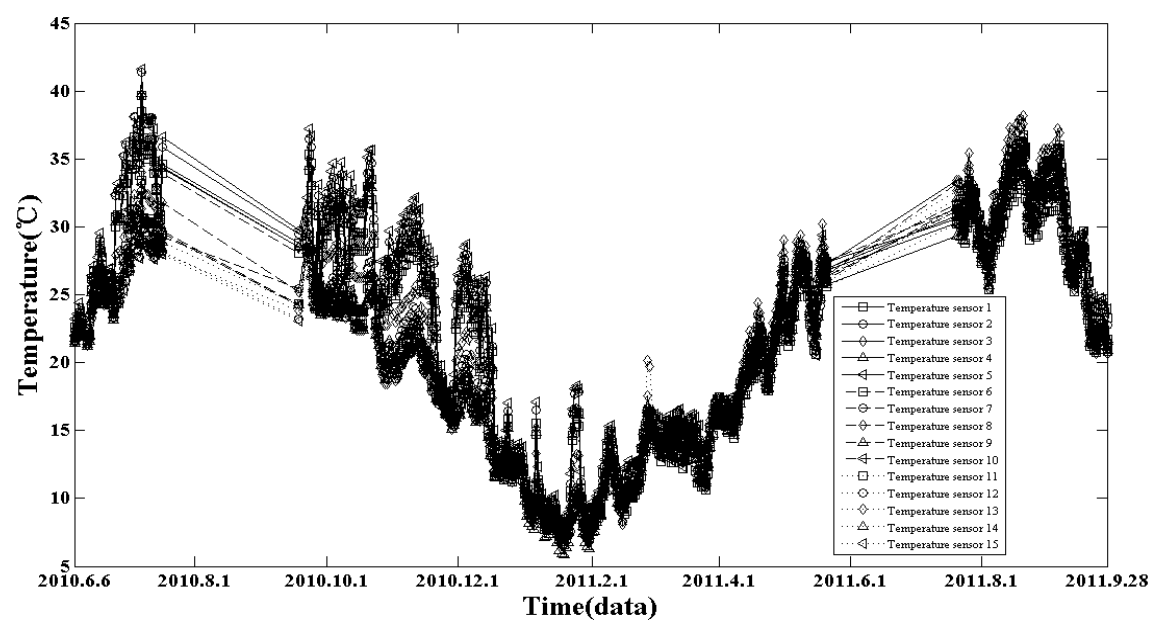

Fig. 5. Temperature change curve of sensors in cable trench and cable pit in 479 days 


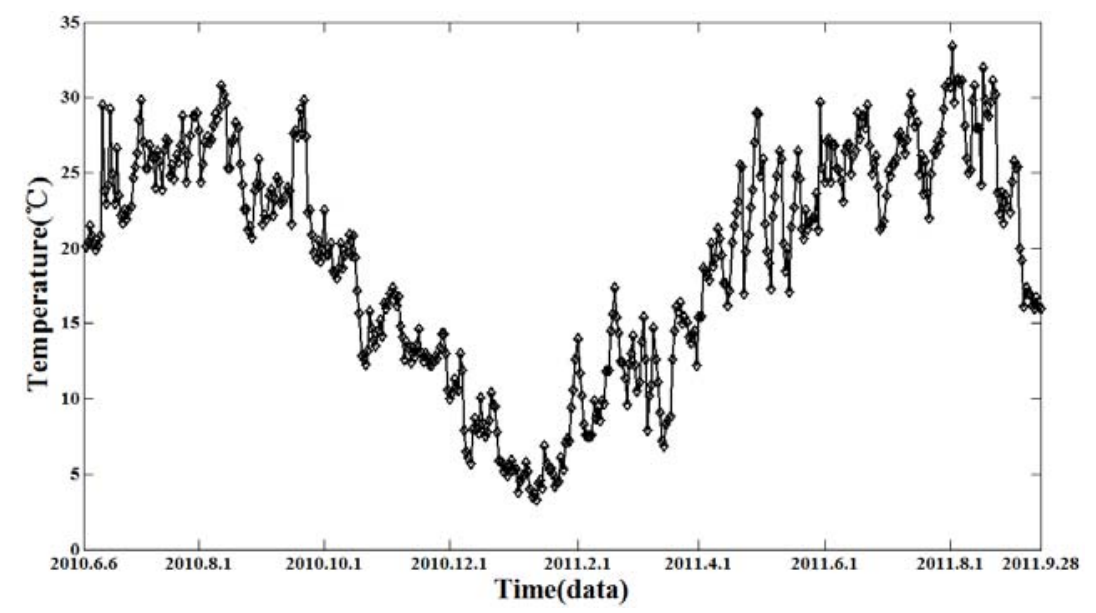

Fig. 6. Temperature change curve of Yanjin environment in 479 days

As shown in Fig. 5, the summarizing of 60 metal armored optical fiber Bragg grating temperature sensor monitoring data, shows that the change trends of the basic temperature curve are consistent, the temperature curves of so many points are similar, there is no special point to deal with. In the long-term monitoring of 479 days, the minimum of the average daily temperature range of 60 sensors is $21.24^{\circ} \mathrm{C}$, detected at number 9 trench 1 , The maximum is $37.43^{\circ} \mathrm{C}$, detected at number 4 trench 2 , which reached its maximum temperature $43.41^{\circ} \mathrm{C}$ on July 3,2010 , and the minimum temperature is $4.37^{\circ} \mathrm{C}$ on January 17 , 2011, detected at number 13 trench 1.

As shown in Fig. 6, the environment temperature on January 19, 2011 reached its lowest $3.31^{\circ} \mathrm{C}$, on October 15, 2011, reached its highest $33.36{ }^{\circ} \mathrm{C}$.

Through the long-term monitoring, we see that the temperature change of metal armored fiber Bragg grating temperature sensor is consistent with the environment temperature change, which is influenced seriously by the season. There is no abnormalities or mutation in the temperature change, the change extent of the maximum and minimum value of temperature of cable trench and cable pit shows they are in normal working condition; According to the scope and the change of the long-term change trend, cable trench and cable pit temperature anomaly alarm function can be enabled.

\section{Conclusion}

This article is about the armored optical fiber Bragg grating metal temperature sensor used in Yanjin substation in cable trench, able Cable pit, real-time online monitoring the operation situation of high voltage cables. Through the comparative analysis and monitoring of Yanjin environment within 24 hours and 479 days of temperature data change curves: cable surface temperature and environmental temperature change trend are consistent; the minimum of the average daily temperature range of 60 sensors is $21.24^{\circ} \mathrm{C}$,detected at number 9 trench 1 , The maximum is $37.43^{\circ} \mathrm{C}$, detected at number 4 trench 2 , which reached its maximum temperature $43.41^{\circ} \mathrm{C}$ on July 3, 2010, and the minimum temperature is 4.37 ${ }^{\circ} \mathrm{C}$ on January 17, 2011,number 13 trench 1. Metal armored fiber Bragg grating temperature sensor temperature changes influenced by seasons; The temperature change has no abnormalities, mutation, the change extent of the maximum and minimum value of temperature of cable trench and cable pit shows they are in normal working condition.

Cable surface temperature of cable and the carrying capacity have a certain relationship, but the cable carrying capacity, influenced by laying, operation conditions and the surrounding environment, is difficult to determine, according to the specific practical problems, we propose specific research contents and methods [7],[8]. Through the optical fiber Bragg grating sensor surface temperature of the cable to calculate the temperature of cable fiber core conductor, and then calculate the ampacities of cables, forecast the maximum load of cable, so that provide the technical basis for the electric power dispatching, to improve the cable of transmission capacity, make the cable work with its greatest potential [9]. 


\section{References}

[1] Zhang YJ, Kang RX, Lou JB, Wei B, Li J, Chen LJ, Su YL. Distributed temperature sensor based on fiber grating of cable. Laser \& Infrared, 2010; 40(4):405-409.

[2] Li J. Application of optical fiber grating temperature sensing system to on-line monitoring for power cables. East China Electric Power, 2005; 33(12):61-63. (in Chinese)

[3] Peng C, Zhao JK, Miao FG. Distributed temperature system applied in cable temperature measurement. High Voltage Engineering, 2006; 32(8):43-45.

[4] Zhu Y, Fu YM, Chen WM. Online deflection monitoring system for dafosi cable-stayed bridge. Journal of Intelligent Material Systems and Structures, 2006; 17:(8/9).

[5] Li C, Zhang YM, Zhao YG, Li LJ, Fiber Grating: Principles, Techniques, and Applications, Beijing: Science Press; 2005.

[6] Li C, You J, et al. Two-pipe fiber Bragg grating temperature sensor. Optical Technique, 2010: 36(2):1-4.

[7] Fan YB, Zhang L, Meng SX, et al. Calculation of current rating for medium and low medium and low voltage XLPE cable in cluster laying. High Voltage Engineering, 2005; 31(10):59-60.

[8] Zeng DX. A study of current-carrying capacity of drawlead cable in condenser bushing. IEEE Trans. on Power Delivery, 2000; 15(3):925-930.

[9] Liu YG, Luo JH. Mathematical method of temperature calculation of power cable reachedor in real time. High Voltage Engineering, 2005; 31(5):52-54. 